\title{
Direct and correlated responses to selection on J HE activity in adult and juvenile Gryllus assimilis: implications for stage-specific evolution of insect endocrine traits
}

\author{
ANTHONY J . ZERA*, TRAVIS SANGER \& GRETCHEN L. CISPER \\ School of Biological Sciences, University of Nebraska, Lincoln, NB 68588, U.S.A.
}

\begin{abstract}
Two-way selection on the activity of the endocrine regulator, juvenile hormone esterase (JHE), in adults of the cricket, Gryllus assimilis, resulted in a realized JHE heritability of $0.059 \pm 0.001$. This heritability was significantly lower than the realized JHE heritability measured previously during selection on the last juvenile stadium $(0.26 \pm 0.04)$. We observed strong correlated responses to selection on JHE activity during different periods of adulthood but very weak correlated responses in the last juvenile stadium. The additive genetic correlation between JHE activities in adults and juveniles, measured from the two pairs of correlated and direct responses obtained from the present adult and the previous juvenile selection studies, did not differ significantly from zero. Lines from the earlier juvenile selection study, reassayed for correlated responses after an additional five generations of selection, exhibited only weak correlated responses for JHE activity in adult and penultimate stages. Results from the present adult and the previous juvenile selection studies provide a consistent picture of strong withinstage JHE correlated responses in either adult or juvenile stages. By contrast, only weak JHE correlated responses occur in contiguous developmental stages, irrespective of whether the stages are physiologically or morphologically similar or dissimilar, or whether JHE activity was selected before or after the stage in which the correlated responses were measured. The regulation of a particular life cycle stage by JHE (juvenile development) can be modified by selection without necessarily altering its regulatory role in other stages (adult reproduction).
\end{abstract}

Keywords: genetic correlation, heritability, JH (juvenile hormone), JHE (juvenile hormone esterase).

\section{Introduction}

Although a wealth of information has been obtained on the chemistry, biochemistry, and physiology of insect endocrine traits (Downer \& Laufer, 1983; Kerkut \& Gilbert, 1985; Gupta, 1990), populationgenetic aspects of insect endocrinology remain poorly studied. At issue are the nature and magnitude of genetic variation and covariation for hormonal features (hormone titres, hormone receptors, activities of enzymes that degrade or synthesize hormones) and the relationship between variation in hormonal and whole organism (life history) traits. Fundamental genetic parameters such as heritability and genetic correlation have been measured on only

*Correspondence. E-mail: azera@unlinfo.unl.edu three endocrine traits in one insect species (Zera \& Zhang, 1995; Gu \& Zera, 1996; Zera et al., 1996). Population-genetic data are available for only a few other cases of endocrine variation in insects (Wilson \& Thurston, 1989; Zera \& Tiebel, 1989; Zera et al., 1989). This paucity of population-genetic information not only limits our understanding of the microevolutionary processes that shape the insect endocrine system, it also limits our understanding of the mechanisms underlying the evolution of organismal traits that are regulated hormonally (life history traits).

During the past few years we have been investigating the quantitative genetics of naturally occurring variation for the endocrine trait, juvenile hormone esterase (JHE) activity, in the cricket Gryllus assimilis (Zera \& Zhang, 1995; Gu \& Zera, 
1996; Zera et al., 1996). Our long-term goals are to identify the magnitude and nature of genetic variation and covariation for a 'typical' endocrine regulator. Juvenile hormone esterase is a hydrolytic enzyme which degrades a key developmental and reproductive hormone, juvenile hormone $(\mathrm{JH})$ (Roe \& Venkatesh, 1990). JHE plays an important role in reducing the $\mathrm{JH}$ titre during the last stadium, a period at which the concentration of this hormone must be lowered in order for metamorphosis to proceed (Zera \& Tiebel, 1989; Roe \& Venkatesh, 1990). JHE is also thought to regulate the JH titre during reproduction in some insects (Roe \& Venkatesh, 1990).

Bi-directional selection on JHE activity during the early portion of the last juvenile stadium of $G$. assimilis (the 10-day stage prior to adulthood) resulted in a strong direct response to selection (realized heritability $=0.26 \pm 0.04$ ) and strong correlated responses to selection at different points of the last juvenile stadium (Zera \& Zhang, 1995; Zera et al., 1996). These results indicate that, at least under laboratory conditions, JHE activity has the potential for rapid evolutionary change and JHE activities throughout the entire last stadium evolve as a tightly correlated unit. By contrast, we obtained no evidence for the existence of correlated responses in adults when JHE activity was selected in juveniles. This latter result shows that the regulation of juvenile development by JHE can be modified by selection without necessarily altering its regulation of adult reproduction.

Our previous quantitative-genetic analyses of JHE in $G$. assimilis left a number of important issues either unresolved or unstudied, which we address in the present study. First, our earlier studies focused almost exclusively on JHE variation and covariation during a single stage of development, the last juvenile stadium. These data provide no information concerning the extent to which quantitative-genetic features of JHE during this stage are representative of other periods of development of $G$. assimilis, especially those that differ in physiology and morphology from the last juvenile stadium. To address this issue we undertook a complementary selection experiment in which JHE activity was directly selected in adults and correlated responses were measured in both adults and juveniles. Secondly, although the study of Zera \& Zhang (1995) eliminated the possibility that selection on JHE during the juvenile stage results in strong correlated responses in adults, we could not eliminate the alternate hypothesis that moderate to weak correlated responses exist which require more than five generations of selection to become detectable. To address this issue, we reassayed the lines of the juvenile selection experiment after an additional five generations of selection. Finally, we also measured JHE activities in the high and low selected lines of the juvenile selection study during the penultimate stage [the developmental stage just prior to the last (ultimate) stadium]. This was to determine whether JHE activities exhibit genetic correlations between juvenile stages that are similar in physiology and morphology.

\section{Materials and methods}

Adult selection experiment: source population, rearing conditions and life cycle stages

The source population of $G$. assimilis used for the adult selection experiment was the same as that used in the previous juvenile selection study of Zera \& Zhang (1995). This population was originally derived from 21 impregnated females collected at Homestead, Florida in 1992 and was maintained by continuously breeding 250-300 adults until sampled to produce the founding populations for the present selection study. Juvenile insects were raised in 10 gallon aquaria at $28^{\circ} \mathrm{C}$ under a $16 \mathrm{~h}$ light: $8 \mathrm{~h}$ dark photoperiod and were fed the dry diet described in Zera \& Rankin (1989). Under these conditions, the penultimate juvenile stage (stadium) lasts $\approx 7-8$ days while the morphologically similar last juvenile stadium lasts 9-10 days. During the selection experiment, when adults of known age were required, laststadium crickets were checked every $24 \mathrm{~h}$ for adult moults. Two to eight newly moulted (day 0) adults were placed in $2 \mathrm{~L}$ plastic boxes and were fed the same dry diet as juveniles (Zera \& Rankin, 1989). During the last generation of selection, when enzyme activities were measured on last stadium as well as adult crickets, the same procedures were used to identify newly moulted last-stadium individuals. Additional rearing information can be found in Zera \& Zhang (1995).

\section{Phenotypes selected}

Haemolymph (blood) JHE activity, measured on the sixth day after adult eclosion, was the trait subjected to direct selection. Methods used to bleed crickets and to quantify JHE activity are reported in $\mathrm{Gu} \&$ Zera (1994, 1996). Day 6 was chosen as the age at which to select JHE activity because it was one of the two adult stages at which indirect responses in JHE activity had been measured in the earlier juve- 
nile selection study (Zera \& Zhang, 1995). Obtaining an adult heritability estimate on this day would allow us to test hypotheses which explain the absence of a correlated response in the juvenile selection study (lack of genetic variability) and also would allow us to estimate the additive genetic correlation between adult and juvenile JHE activities (Falconer, 1989; see below). In addition, days 4-6 of adulthood is the period of time at which ovaries first begin extensive growth which is possibly regulated by $\mathrm{JH}$ and possibly JHE.

During the last generation of selection, in each of the selected lines, haemolymph JHE activity was also measured at the following points in development: Days 3, 5 and 8 of the 10-day last juvenile stadium, and Days 2 and 10 of adults. This was done to test for correlated responses to JHE activity in the various adult and juvenile stages. JHE activity exhibits a single peak midway through the last juvenile stadium. Thus Days 3, 5 and 8 of the last stadium correspond to prepeak, peak and postpeak JHE activities during that stage. The adult developmental profile of JHE activity is roughly flat and activity is about 10 -fold lower than that of the peak during the last stadium. Details of the JHE activity developmental profile can be found in $\mathrm{Gu} \&$ Zera (1994) and Zera \& Zhang (1995).

\section{Selection protocol}

The basic experimental design employed in this study was replicated, divergent selection (Falconer, 1989). This method involves selecting upward and downward for some trait without maintaining unselected controls. Each selected line acts as an internal control for the alternate line to eliminate changes caused by environmental variation. The response to selection is measured as the divergence in the mean phenotypic value between the upward and downward selected lines, and the realized heritability estimated from this method is the heritability of divergence between upward and downward selection. This heritability estimate is equal to twice the average heritability for selection in one direction. This method of selection has two important advantages. First, for each replicate selection trial, only two rather than three groups are required. Secondly, for a given sample size, the statistical power is greater using divergent selection compared with a design in which upward and downward selected lines are compared to a common control (Falconer, 1989; Gromko et al., 1991). A limitation of this method is that asymmetrical responses to selection cannot be identified (but see Gromko et al., 1991). JHE activity in the last stadium of $G$. assimilis exhibited a symmetrical response to upward and downward selection (Zera \& Zhang, 1995).

Two separate base populations (blocks) were founded by measuring haemolymph JHE activity on the sixth day of adulthood of 92 (block 1) or 89 (block 2) G. assimilis taken from the source population. In each of these two base populations the 10-11 males and the 10-11 females with highest phenotypic values were chosen as breeders to produce each of the two high-selected lines and the same number of adults with the lowest JHE activities were chosen to form each of the two low-selected lines. Individuals comprising the block 1 base population were sampled during November 1994 whereas those comprising the block 2 base were sampled during January 1995.

During each generation excluding the last, haemolymph JHE activity was measured on 70-100 Day-6 adults from each of the four lines [mean $( \pm$ SEM $)$ number of individuals sampled per generation per line $=92 \pm 5.2]$. As for the base population, the 10-12 females and 10-12 males exhibiting the highest (high-selected lines) or lowest (low-selected lines) JHE activities were chosen as breeders to produce the next generation. Selected adults from a particular line were bred together in a single aquarium containing food, water and oviposition material. JHE activity was selected for four (block 1) or five (block 2) generations.

\section{Estimation of heritabilities and coefficients of additive genetic and residual variation}

In each of the two blocks, realized heritabilities were estimated in the standard manner by linear regression of the cumulative response in mean JHE activity each generation vs. the cumulative selection differential (Falconer, 1989). The cumulative response for a particular block was the difference between the mean JHE activity in the high vs. the low line during a particular generation. The cumulative differential for each block was the difference between the mean JHE activity of selected high breeders and mean JHE activity for the total highselected population plus the difference between JHE activity of the low breeders compared with the total low-selected population summed over all generations. The realized heritabilities of divergence for each block were divided by two and averaged to estimate the average realized heritability in one direction. The standard error reported with the heritability estimate is the empirical standard error derived from the variance in heritabilities between 
the two blocks (Hill, 1971). Because generation means computed from untransformed activities were correlated with their sample variances, regressions were performed on log-transformed activities.

Coefficients of additive genetic variation $\left(\mathrm{CV}_{\mathrm{A}}\right.$; standardized additive genetic variability; Houle, 1992; Messina, 1993) for JHE activity were computed as follows: $C V_{\mathrm{A}}=\left(100 \sqrt{V_{\mathrm{A}}}\right) / \mathrm{d}$, where $V_{\mathrm{A}}$ is the additive genetic variance and is the mean JHE activity in the base population. $V_{\mathrm{A}}$ was computed in the standard fashion as follows: $V_{\mathrm{A}}=h^{2} V_{\mathrm{P}}$, where $h^{2}=$ realized heritability (estimated from the selection experiment) and $V_{\mathrm{P}}$ is the phenotypic variance of JHE activity in the base population. The coefficient of residual variation $\left(C V_{\mathrm{R}}\right)$, which is a standardized measure of components of the phenotypic variance attributable to environmental variance plus all nonadditive genetic components, was computed as follows: $C V_{\mathrm{R}}=\left(100 \sqrt{V_{\mathrm{P}}-V_{\mathrm{A}}}\right) /$.

\section{Correlated responses in the adult selection experiment}

Indirect responses in lines selected for high or low JHE activity on Day 6 of adulthood were measured on Days 2 and 10 of adults (within-stage correlated response) and on days 3,5 and 8 of the last stadium (between-stage correlated response). JHE activity differences between lines were tested separately in each block on each of these five days by singleclassification ANOVA. Since the tests were performed on two independent selection trials (blocks), probabilities were multiplied by 2 to adjust for experiment-wide error (Dunn, 1959).

\section{Correlated responses in the juvenile selection experiment}

We continued the juvenile selection study of Zera \& Zhang (1995), which involves direct selection on JHE activity on the third day of the 10-day last juvenile stadium. JHE activities were compared between high and low-selected lines in each of the three blocks on Days 2 and 6 of adulthood during the tenth generation of selection. We also tested for the existence of correlated responses in the penultimate stadium during the seventh generation of selection by comparing JHE activities between the high- and low-selected lines of two of the three blocks. As in the adult selection experiment, tests for correlated responses consisted of separate singleclassification ANOVAS of JHE activities in high- and low-selected lines on each day of each block studied. To adjust for experiment-wide error, probabilities from these ANOVAS were multiplied by the number of independent selection trials (blocks) studied.

Additive genetic correlation between adult and juvenile J HE activities

In our complementary selection experiments, JHE activity on Day 3 of the last juvenile stadium exhibited a direct response under juvenile selection and a correlated response under adult selection. The reverse situation occurred for JHE activity in Day-6 adults. The two direct and two correlated responses were used to estimate the additive genetic correlation between the adult and juvenile JHE activities on these days using eqn (19.7) from Falconer (1989): $r_{\mathrm{A}}^{2}=\left(C R_{\mathrm{y}} / R_{\mathrm{x}}\right)\left(C R_{\mathrm{x}} / R_{\mathrm{y}}\right)$ where $r_{\mathrm{A}}^{2}$ is the square of the additive genetic correlation, and $R$ and $C R$ are the direct and correlated responses for trait $\mathrm{X}$ (JHE activity in juveniles) and trait Y (JHE activity in adults). As for the direct response (see above), the correlated response was calculated as the difference between JHE activities in the high- and low-selected lines at the age not directly selected. Direct and correlated responses used to estimate $r_{\mathrm{A}}$ were measured on generation 10 (all blocks of juvenile selection), generation 4 (block 1 of adult selection), or generation 5 (block 2 of adult selection). Replicate estimates of $r_{\mathrm{A}}^{2}$ were obtained by two different methods. In method $\mathrm{A}$, the two $C R / R$ ratios obtained by adult selection (one from each block) were averaged and multiplied by each of the three $C R / R$ ratios obtained by juvenile selection. This resulted in three estimates of $r^{2}{ }_{\mathrm{A}}$. In method $\mathrm{B}$, the three $C R / R$ ratios obtained by juvenile selection were averaged and multiplied by each of the two $C R / R$ ratios obtained by adult selection. This yielded two estimates of $r_{\mathrm{A}}^{2}$. Additive genetic correlations were obtained by taking the square root of the various $r_{\mathrm{A}}^{2}$ estimates.

\section{Results}

Adult selection: response to selection, heritability and coefficients of additive genetic and residual variation for J HE activity

JHE activity in adult $G$. assimilis responded rapidly to bi-directional selection with mean activities differing significantly between the high and low lines of each block after only one generation of selection (Fig. 1; ANOVAs: block 1: $F_{1,201}=8.07, P<0.006$; block 2: $\left.F_{1,209}=4.75, P<0.05\right)$. By the last generation of selection, highly significant differences were observed between the high- and low-selected lines of each block (Fig. 1; ANOVAs: block 1: $F_{1,36}=21.6$, 

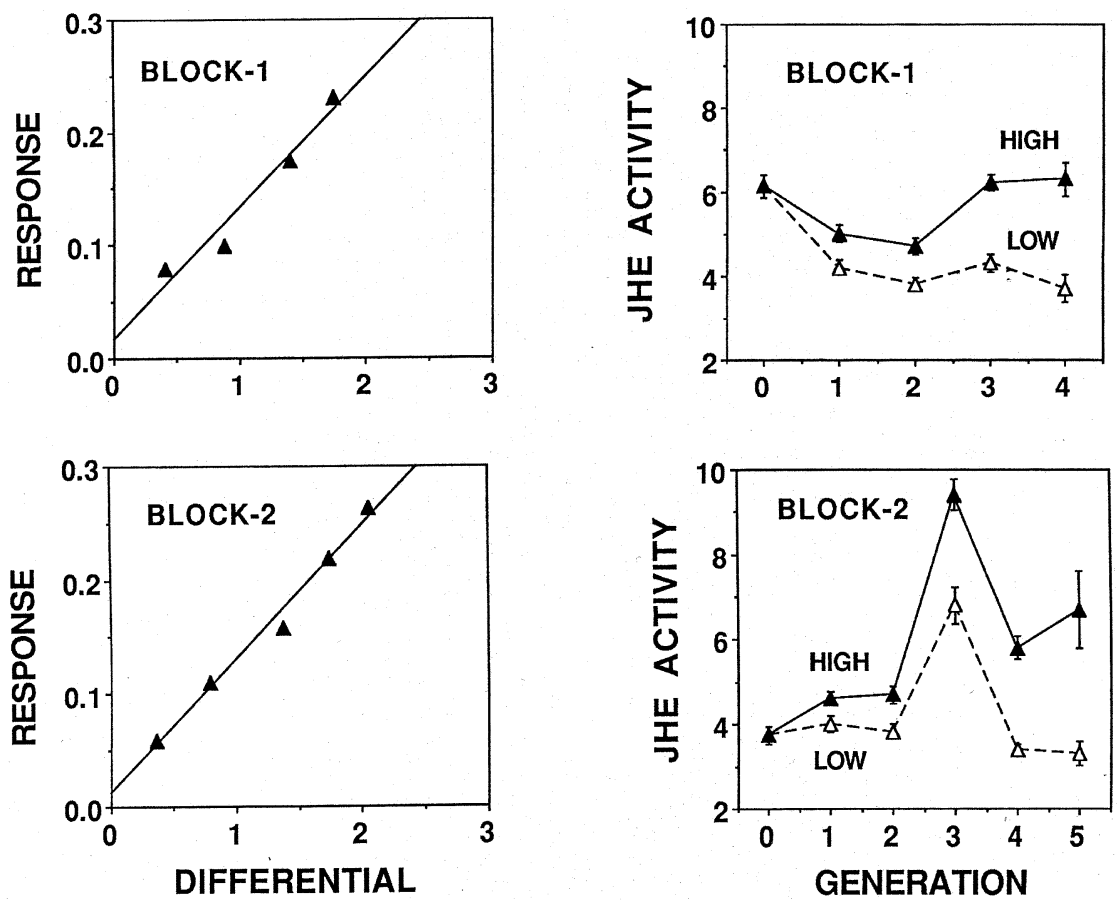

Fig. 1 Mean $( \pm$ SEM) haemolymph JHE activities (right panels) and selection responses and differentials (left panels) during the course of bi-directional selection on day- 6 adult Gryllus assimilis. Upper and lower panels depict selection on block 1 and block 2 lines, respectively. JHE activities are in units of nmol $\mathrm{JH}$ acid produced $/ \mathrm{min} / \mathrm{mL}$ haemolymph. See Methods for additional details.

$P<0.001$; block 2: $\left.F_{1,33}=10.6, P<0.005\right)$. For each block, linear regression of the cumulative selection response vs. the cumulative differential resulted in a significant slope (realized heritability of divergence) (Fig. 1; Table 1). The average realized heritability for JHE activity in one direction (slope/2) for each block and the average across blocks are given in Table 1 . The overall average $(0.059 \pm 0.001)$ is signi- ficantly different from zero $\left(t_{1}=118 ; P<0.01\right)$ indicating a significant heritability for haemolymph JHE activity in day- 6 adults. Means and phenotypic variances for JHE activity in the base population of each block and the coefficients of additive genetic and residual variation are given in Table 1. For comparison (see Discussion), corresponding statistics on JHE activities derived from the previous

Table 1 Means and phenotypic variances for haemolymph JHE activity in the base populations and realized heritabilities, coefficients of additive genetic variation $\left(C V_{\mathrm{A}}\right)$ and coefficients of residual variation $\left(C V_{\mathrm{R}}\right)$ after bi-directional selection in adult or juvenile Gryllus assimilis

\section{Study}

\begin{tabular}{lcc}
\hline \multicolumn{2}{c}{ Adult selection* } & \\
\hline Block 1 & Block 2 & Juvenile selection $\dagger$ \\
\hline \multirow{2}{*}{6.13} & 3.74 & $31.33 \S$ \\
6.97 & 4.12 & 150.02 \\
0.058 & 0.059 & \\
& $0.06 \pm 0.001(N=2) \uparrow$ & $0.26 \pm 0.04(N=3)$ ฯ \\
& $11.8 \pm 1.41(N=2)$ & $20.12 \pm 1.15(N=3)$ \\
& $46.4 \pm 4.57(N=2)$ & $33.5 \pm 0.68(N=3)$ \\
\hline
\end{tabular}

*Present study.

$\dagger$ Study of Zera \& Zhang (1995).

$\ddagger$ nmol JH hydrolysed/min/mL haemolymph.

§The juvenile selection study (Zera \& Zhang, 1995) involved one base population from which all lines were derived.

IN $=$ number of replicate selection trials (blocks). 
juvenile selection study of Zera \& Zhang (1995) are also given in Table 1.

Adult selection: correlated responses in adults and juveniles

Developmental profiles of JHE activity on Days 2, 6 and 10 of adults for the two pairs of high- and low-selected lines measured during the last generation of selection are presented in Fig. 2. Visual examination of these profiles and results of singleclassification ANOVAS indicate consistently higher JHE activities in high- vs. low-selected lines in each block on all adult ages. JHE activity was significantly higher in the high- vs. low-selected line on Day 2 in each block (block 1: $F_{1,48}=13.4, P<0.002$; block 2: $\left.F_{1,32}=11.0, P<0.01\right)$. On day 10 , JHE activity was significantly higher in the high vs. low selected line in block $2\left(F_{1,13}=8.18, P<0.04\right)$ and was nearly significant in block $1\left(F_{1,35}=4.88,0.06<P<0.08\right)$. We conclude from these results that significant positive correlated responses for JHE activity exist on Days 2 and 10 in adults.

Mean $( \pm$ SEM) JHE activities in the high- and low-selected lines on Days 3, 5 and 8 of the last juvenile stadium measured during the last generation of adult selection are presented in Fig. 2. As was the case for JHE activities in adults, JHE activities in juveniles were consistently higher in the high- vs. low-selected lines in each block on each day studied. However, the magnitude of the differences between lines was much less in juveniles than in adults. JHE activities only differed significantly between high and low lines in block 1 on Day 3 $\left(F_{1,48}=16.6, P<0.002\right)$. The five other AnOvas were nonsignificant (block 2, Day-3: $F_{1,34}=0.42, P>0.1$; block 1, Day 5: $F_{1,35}=3.69, P>0.1$ or block 2, Day 5: $F_{1,40}=1.16, P>0.1$; block 1, Day 8: $F_{1,46}=1.10$, $P>0.1$ or block 2, Day 8: $F_{1,27}=4.09, P>0.1$ ). Despite the nonsignificance of most of these individual ANOvAs, the higher mean JHE activity in the high vs. low line on each day in each block (six comparisons) indicates a significantly higher JHE activity in high-selected lines during the last stadium taken as a whole (sign test: $P=0.016$ ). These results show the existence of weak, positive correlated responses to selection during the last juvenile stage.

\section{J uvenile selection: correlated responses in adults and juveniles}

Mean JHE activities in Day-2 and Day-6 adults after 10 generations of selection on JHE activity in the last juvenile stadium are given in Table 2. For comparative purposes, the direct responses are also given in Table 2. JHE activity in adults was higher in high- vs. low-selected lines of block 2 on both Day 2 $\left(F_{1,30}=77.5, \quad P<0.001\right)$ and Day $6 \quad\left(F_{1,31}=19.8\right.$,
Fig. 2 JHE activities in high- and low-selected lines of Gryllus assimilis. Panels (a) and (b) contain adult activities and panels (c) and (d) contain activities of last-stadium crickets. Activities are expressed in the same units as those in Fig. 1 and were measured during the last generation of selection on adults.
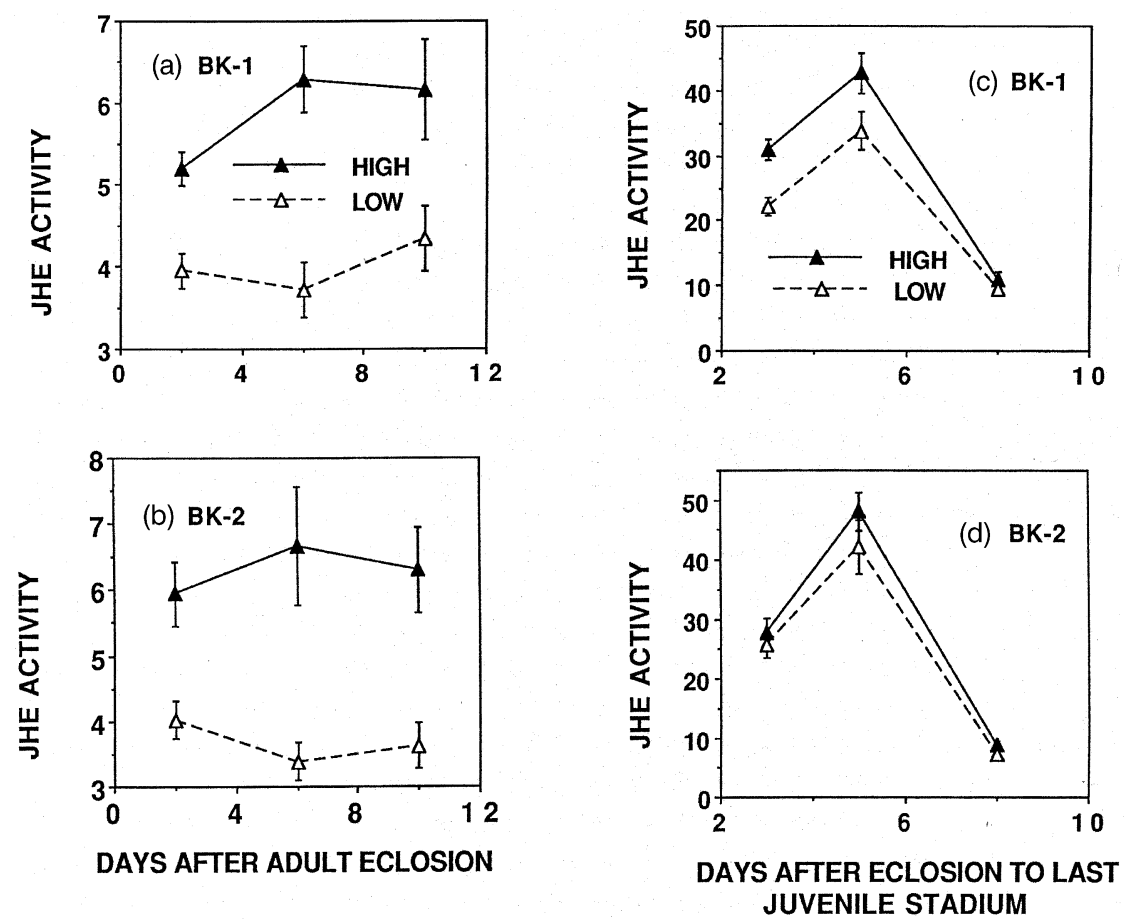
Table 2 Direct and correlated responses to selection on haemolymph juvenile hormone esterase activity in G. assimilis

\begin{tabular}{llcc}
\hline & & \multicolumn{2}{c}{ Indirect response $\dagger$} \\
\cline { 3 - 4 } Line/Block & Direct response* & Day 2 & Day 6 \\
\hline H-1 $\ddagger$ & $44.9 \pm 1.18(84 \S)$ & $3.48 \pm 0.34(15)$ & $2.98 \pm 0.34(16)$ \\
L-1 & $4.44 \pm 0.28(81)$ & $2.81 \pm 0.32(16)$ & $4.41 \pm 0.61(15)$ \\
H-2 & $48.4 \pm 1.63(88)$ & $6.47 \pm 0.52(16)$ & $3.67 \pm 0.03(16)$ \\
L-2 & $7.55 \pm 0.41(82)$ & $1.73 \pm 0.13(16)$ & $2.12 \pm 0.18(17)$ \\
H-3 & $53.6 \pm 1.48(83)$ & $4.53 \pm 0.28(16)$ & $4.39 \pm 0.42(16)$ \\
L-3 & $8.89 \pm 0.50(80)$ & $3.39 \pm 0.18(16)$ & $2.98 \pm 0.22(16)$ \\
\hline
\end{tabular}

*Day 3 of the last juvenile stadium (generation 10).

$\dagger$ Day after the adult moult (generation 10).

$\ddagger \mathrm{H}=$ high selected, $\mathrm{L}=$ low selected; the number following the letter refers to the block (independent selection trial; see Zera \& Zhang, 1995).

$\S$ Values are mean JHE activities ( $\mathrm{nmol} / \mathrm{min} / \mathrm{mL}$ haemolymph) followed by \pm standard error and sample size (in parentheses).

$P<0.005)$. Similar results were obtained for block 3 lines (Day 2, high vs. low: $F_{1,31}=10.7, P<0.01$; Day 6, high vs. low: $F_{1,30}=8.70, P<0.03$ ) However, in block 1, adult JHE activities did not differ significantly between high- and low-selected lines on either Day $2\left(F_{1,29}=2.03, P>0.1\right)$ or Day $6\left(F_{1,29}=4.30\right.$, $P>0.1)$. These results show the existence of positive correlated responses to selection in adults in blocks 2 and 3 but not in block 1 .

We also observed significant positive correlated responses in penultimate-stadium $G$. assimilis in both blocks 1 and 2 during the seventh generation of selection on JHE activity in last-stadium crickets. Correlated responses were not measured in block 3 . Mean $( \pm$ SEM) JHE activity $(\mathrm{nmol} / \mathrm{min} / \mathrm{mL})$ was significantly higher in the high- vs. the low-selected line of block 1 (high $=7.3 \pm 0.6$, low $=5.1 \pm 0.3$; ANOVA: $\left.\quad F_{1,16}=10.7, \quad P<0.01\right)$ and block 2 $\left(\right.$ high $=6.5 \pm 0.4$, low $=4.3 \pm 0.4 ;$ ANOvA: $F_{1,16}=13.0$, $P<0.01)$. Each of these means was based on JHE activities of nine crickets.

\section{Additive genetic correlations between adults and juveniles}

Individual and mean $( \pm \mathrm{SEM})$ additive genetic correlations between JHE activities on Day 6 of adult and Day 3 of last-stadium G. assimilis obtained by method A (see Materials and methods) are as follows: -0.28 (block 1), 0.29 (block 2), 0.26 (block 3 ), and $0.09 \pm 0.18$. Analogous values obtained by method B are as follows: 0.20 (block 1), 0.11 (block 2 ), and $0.16 \pm 0.04$. In neither case did the mean additive genetic correlation differ significantly from zero ( $t$-tests; $P>0.1$ ).

\section{Discussion}

The present study is the first quantitative-genetic analysis of naturally occurring endocrine variation in an adult insect. This study both corroborates and extends the results obtained in our previous quantitative-genetic analyses of JHE variation in juvenile G. assimilis (Zera \& Zhang, 1995; Gu \& Zera, 1996; Zera et al., 1996). Three important points emerge from these studies of JHE variation in adults and juveniles. First, JHE activity exhibits sufficient additive genetic variance to respond to selection in all developmental stages that were studied. Secondly, strong within-stage JHE genetic correlations exist in both adult and juvenile stages. Thirdly, JHE activities exhibit only weak genetic correlations between different life-cycle stages. Thus, alteration of JHE activity in any one developmental stage by selection results in only minimal changes in JHE activity in other developmentally contiguous stages.

\section{Heritabilities}

Although JHE activity exhibited a significant realized heritability in both adult and juvenile $G$. assimilis, the magnitude of the adult JHE heritability was only one-quarter that measured in juveniles (Table 1; ANOvA of log-transformed heritabilities: $\left.F_{1,3}=100.4, P<0.005\right)$. The JHE heritability in adults also is only one-sixth that of the heritability for JH-binding activity in juvenile G. assimilis ( $\mathrm{Gu} \&$ Zera, 1996), the only other endocrine trait for which a heritability estimate is available, and is significantly lower than nearly all heritabilities of enzyme activities in Drosophila melanogaster (Clark, 1990).

(C) The Genetical Society of Great Britain, Heredity, 80, 300-309. 
The significance, if any, of the low JHE heritability in adult $G$. assimilis is unknown. The low adult JHE heritability has two important consequences for the evolution of JHE activity in G. assimilis. First, JHE activity evolves at a considerably slower rate in adults compared with last-stadium juveniles under the same selection intensity (compare Fig. 1 of the present study with fig. 1 of Zera \& Zhang, 1995). Secondly, because correlated responses are dependent upon the heritabilities of the traits directly and indirectly selected (Falconer, 1989), the low adult heritability of JHE activity also limits the correlated responses in adults or juveniles when selection is applied to the alternate stage (Fig. 2; Table 2).

Recently, Houle (1992) and Messina (1993) have advocated using direct standardized measures of additive genetic variance (coefficient of additive genetic variance, $C V_{\mathrm{A}}$ ) and residual variance (coefficient of residual variance, $C V_{\mathrm{R}}$ ) to assess differences in genetic variability between traits (see Methods for definitions and calculation of $C V_{\mathrm{A}}$ and $C V_{\mathrm{R}}$ ). Comparisons of $C V_{\mathrm{A}}$ and $C V_{\mathrm{R}}$ for adult vs. juvenile JHE activities in $G$. assimilis (Table 1) indicate that the lower JHE heritability in adults is primarily caused by a reduction in additive genetic variance and to a lesser degree by increased residual variance. $C V_{\mathrm{A}} \mathrm{s}$ and $C V_{\mathrm{R}} \mathrm{s}$ for $\mathrm{JHE}$ activities are similar to values for fitness traits and are higher than values for morphological traits in Drosophila and Callosobruchus (Houle, 1992; Messina, 1993).

\section{J HE correlations within life cycle stages}

The pattern of strong, within-stage correlated responses for JHE activities, observed in the present study (Fig. 2), is similar to the pattern obtained in our previous juvenile selection study. When JHE was selected on Day 3 of the last juvenile stadium (Zera \& Zhang, 1995), correlated responses were observed on two other days of that stadium which were equivalent in magnitude to the direct response. Similarly, in the present adult selection study, the ratio of JHE activity in high- vs. low-selected lines averaged across the two blocks (average divergence between lines) on either Day $2(1.40 \pm 0.08, N=2)$ or Day $10(1.62 \pm 0.18, N=2)$ was very similar to the ratio on Day 6 (direct response; $1.80 \pm 0.1$ ). The similarity between the ratios for the direct and indirect responses and the similarity of the adult JHE developmental profiles for the high- and low-selected lines within each block (Fig. 2) clearly show that the entire JHE activity profile in adults evolves as a correlated unit, as is the case for JHE activities in last-stadium juveniles (Zera \& Zhang, 1995). The strong, positive additive genetic correlations between JHE activities on different days of the last juvenile stadium, observed in a previous half-sib analysis of JHE variation in a different population of G. assimilis ( $\mathrm{Gu} \&$ Zera, 1996), is consistent with this pattern. Thus, the collective results from three separate quantitative-genetic studies indicate that strong within-stage correlated responses or withinstage additive-genetic correlations appear to be a general feature of JHE variation in different life cycle stages of $G$. assimilis. The strong additivegenetic correlations between JHE activity and juvenile hormone binding (JHB) activity on different days of the last stadium of $G$. assimilis ( $\mathrm{Gu} \&$ Zera, 1996; Zera et al., 1996) suggest that positive genetic correlations may be a common feature of endocrine traits that regulate haemolymph juvenile hormone levels in G. assimilis. This is consistent with data on genetic correlations between enzymes involved in intermediary metabolism in Drosophila. Strong correlations have been observed between activities of enzymes that share common substrates, cofactors or subcellular locations (Clark, 1990; Wilton et al., 1992).

\section{J HE correlations between life cycle stages}

In both the adult and juvenile selection studies, we obtained consistent evidence for no more than weak correlated responses for JHE activity in adults or last-stadium juveniles when JHE activity was selected in the alternate stage (Table 2; Fig. 2). Similarly, we also observed only weak correlated responses for JHE activity in the penultimate stadium when JHE activity was selected in the last juvenile stadium (Results). Between-stage correlated responses were barely detected in each of these cases, even after many generations of selection. Furthermore, the additive genetic correlation between JHE activities in adults and last-stadium juveniles, estimated from these correlated responses, did not differ significantly from zero (Results). By contrast, direct responses were detected in each selection study after only one generation (Fig. 1; Zera \& Zhang, 1995). These data collectively show that only weak correlated responses for JHE activity exist between contiguous developmental stages in $G$. assimilis irrespective of whether the stages are physiologically and morphologically similar (penultimate and last juvenile stadia) or dissimilar (last juvenile stadium and adults), or of whether JHE activity 
was directly selected in the developmental stage before or after the stage in which the correlated responses were measured.

Information from two congeners, Gryllus rubens and $G$. firmus, suggest that the patterns of weak between-stage JHE correlations coupled with strong within-stage JHE correlations, observed in our selection studies of G. assimilis (Zera \& Zhang, 1995; present study), may be common in Gryllus species. Genetic stocks of both G. firmus and G. rubens, selected for long or short wings, differ from 2- to 5-fold in JHE activity throughout the last juvenile stadium. However, these stocks exhibit no or only minor differences in JHE activity during the adult stage (Zera \& Tiebel, 1989; Zera et al., 1993; Y. Huang and A. J. Zera, unpubl. data). Thus, stockspecific developmental profiles for JHE activity in these two species are very similar to the profiles obtained when JHE activity was selected during the last juvenile stadium in G. assimilis (Zera \& Zhang, 1995; Table 2).

\section{Mechanistic basis of the genetic correlations and evolutionary implications}

The pattern of strong within-stage correlations coupled with weak between-stage correlations for JHE activity in $G$. assimilis raises many intriguing questions concerning the proximate mechanisms responsible for these patterns and their evolution. The pattern of JHE correlations is consistent with the action of 'temporal loci' (sensu Paigen, 1979), that is, loci whose alleles vary in temporal and spatial expression leading to genotype-specific differences in the developmental appearance and/or tissue distribution of JHE. We have previously documented dramatic differences in the tissue distribution of JHE activity in high- and low-activity lines from the juvenile selection experiment (Zera et al., 1996). In the high-activity lines $\approx 70$ per cent of whole-organism JHE activity occurs in the haemolymph compartment whereas the corresponding value for the low-selected lines is 29 per cent. We have identified no kinetic or thermostability differences among the JHE enzymes from high- and low-selected lines (Zera \& Zeisset, 1996). This further implicates variation in 'regulatory' loci affecting the synthesis, degradation or activation of JHE activity as the major cause of the JHE activity differences between the selected lines. At present we have no information concerning the identity or number of these temporal loci or their mechanism of action. At the very least, the existence of strong
JHE correlations that are restricted to individual stadia shows that the regulation of JHE must be sufficiently variable between adults and juveniles and between different juvenile stadia such that individual loci influencing JHE regulation during one stage have no major effect on JHE regulation during other stages. Little is known about the physiological regulation of JHE except that cephalic neuroendocrine factors and $\mathrm{JH}$ itself appear to be involved in JHE regulation in some moth species (Roe \& Venkatesh, 1990). Even less is known about the regulation of JHE at the molecular level. We are currently focusing on the physiological and molecular regulation of JHE in $G$. assimilis with the ultimate goal of identifying the nature of the temporal loci regulating this enzyme.

There is currently very little information on patterns of genetic correlations between biochemical or physiological traits during development. Thus it is unclear whether the pattern of strong correlations within stadia coupled with weak correlations between stages is unique to $\mathrm{JH}$-regulating factors such as JHE, or is a common feature of endocrine factors or enzymes in general. $\mathrm{JH}$ is a dual-function hormone whose major roles are the regulation of adult reproduction and juvenile development. Since these major roles are developmentally separated, it is not surprising to find also only weak genetic correlations between adult and juvenile stages for a JH-regulator such as haemolymph JHE activity. However, JHE-correlated responses were also as weak between the two juvenile stadia studied (last and penultimate) as they were between juvenile and adult stages. The few studies of between-stage genetic correlations for enzyme activity in Drosophila melanogaster have found no consistent patterns. Some enzymes such as glucose-6-phosphate dehydrogenase do not exhibit genetic correlations between adult and juvenile stages whereas other enzymes, such as alcohol dehydrogenase and catalase, exhibit strong correlations (Miyashita \& Laurie-Ahlberg, 1986 and references therein). Finally, Loeschcke \& Krebs (1996) failed to detect realized genetic correlations between heat-shock resistance in adult and juvenile stages of Drosophila buzzatii. Increased heat-shock resistance evolved in adult or juvenile stages of this species under artificial selection without affecting heat-shock resistance in the alternate stage.

One of the most important but unsolved issues confronting evolutionary biology is the mechanisms underlying the evolution of gene regulation in natural populations. There are very few empirical data on this issue in general and no information on 
the specific question of the microevolution of hormonal regulation. The JHE-selected lines offer considerable promise as tools for investigating the microevolutionary change in gene regulation of an enzyme which plays a key role in the hormonal regulation of development and reproduction.

\section{Acknow ledgements}

Research reported here was supported by NSF grants IBN-9105257 and IBN-95-07388 to A.J.Z.

\section{References}

ClARK, A. G. 1990. Genetic components of variation in energy storage in Drosophila melanogaster. Evolution, 44, 637-650.

DOWNER, R. G. H. AND LAUfER, H. (eds) 1983. Endocrinology of Insects. Alan R. Liss, New York.

DunN, O. J. 1959. Confidence intervals for the means of dependent, normally distributed variables. J. Am. Stat. Ass., 54, 613-621.

FALCONER, D. S. 1989. Introduction to Quantitative Genetics, 3rd edn. Longman Scientific and Technical, New York.

GROMKO, M. H., BRIOT, A., JENSEN, S. C. AND FUKUI, H. H. 1991. Selection on copulation duration in Drosophila melanogaster: predictability of direct response vs. unpredictability of correlated response. Evolution, 45, 69-81.

GU, X. AND ZERA, A. J. 1994. Developmental profiles and characteristics of hemolymph juvenile hormone esterase, general esterase and juvenile hormone binding in the cricket, Gryllus assimilis. Comp. Biochem. Physiol., 107B, 553-560.

GU, X. AND ZERA, A. J. 1996. Quantitative genetics of juvenile hormone esterase, juvenile hormone binding and general esterase activity in the cricket, Gryllus assimilis. Heredity, 76, 136-142.

GuptA, A. P. (ed) 1990. Morphogenetic Hormones of Arthropods, vols 1, 2 and 3. Rutgers University Press, New Brunswick, NJ.

HILL, W. G. 1971. Design and efficiency of selection experiments for estimating genetic parameters. Biometrics, 27, 293-311.

HOULE, D. 1992. Comparing evolvability and variability of quantitative traits. Genetics, 130, 195-204.

Kerkut, G. A. AND GILBERT, L. I. (eds) 1985. Comprehensive Insect Biochemistry, Physiology and Pharmacology, vols 7 and 8: Endocrinology. Pergamon, New York.

LOESCHCKE, V. AND KREBS, R. A. 1996. Selection for heatshock resistance in larval and adult Drosophila buzzatii: comparing direct and indirect responses. Evolution, 50, 2354-2359.

MESSINA, F. A. 1993. Heritability and evolvability of fitness components in Callosobruchus maculatus. Heredity, 71, 623-629.

MIYASHITA, N. AND LAURIE-AHLBERG, c. C. 1986. Developmental variation in effects of the second and third chromosomes on the activities of the glucose-6-phosphate and 6-phosphoguconate dehydrogenases in Drosophila melanogaster. Biochem. Genet., 24, 447-467.

PAIGEN, K. 1979. Genetic factors in developmental regulation. In: Scandalios, J. G. (ed.) Physiological Genetics, pp. 1-61. Academic Press, New York.

ROE, R. M. AND VENKATESH, K. 1990. Metabolism of juvenile hormones: degradation and titer regulation. In: Gupta, A. P. (ed.) Morphogenetic Hormones of Arthropods, pp. 126-179. Rutgers University Press, New Brunswick, NJ.

WILSON, T. G. AND THURSTON, J. 1989. Genetic variation for methoprene resistance in Drosophila melanogaster. J. Insect Physiol., 34, 305-308.

Wilton, A. N., LAURIE-AhlberG, C. C., EMigh, T. H. AND CURTSINGER, J. W. 1982. Naturally occurring enzyme activity variation in Drosophila melanogaster. II. Relationships among enzymes. Genetics, 102, 207-221.

ZERA, A. J. AND RANKIN, M. A. 1989. Wing dimorphism in Gryllus rubens: genetic basis of morph determination and fertility differences between morphs. Oecologia, 80, 249-255.

ZERA, A. J. AND TIEBEL, K. C. 1989. Differences in juvenile hormone esterase activity between presumptive macropterous and brachypterous Gryllus rubens: implications for the hormonal control of wing polymorphism. J. Insect Physiol., 35, 7-17.

ZERA, A. J. AND ZEISSET, M. 1996. Biochemical characterization of juvenile hormone esterases from lines selected for high or low enzyme activity in Gryllus assimilis. Biochem. Genet., 34, 421-435.

ZERA, A. J. AND ZHANG, C. 1995. Direct and correlated responses to selection on hemolymph juvenile hormone esterase activity in Gryllus assimilis. Genetics, 141, 1125-1134.

ZERA, A. J., STRAMBI, C., TIEBEl, K. C., STRAMBI, A. AND RANKIN, M. A. 1989. Juvenile hormone and ecdysteroid titers during critical periods of wing morph determination in Gryllus rubens. J. Insect Physiol., 35, 501-511.

ZERA, A. J. BORCHER, C. A. AND GAINES, S. B. 1993. Juvenile hormone degradation in adult wing morphs of the cricket, Gryllus rubens. J. Insect Physiol., 39, 845-856.

ZERA, A. J., SALL, J. AND SCHWARTZ, R. 1996. Artificial selection on JHE activity in Gryllus assimilis: nature of activity differences between lines and effect on $\mathrm{JH}$ binding and metabolism. Arch. Insect Biochem. Physiol., 32, 421-428. 